\title{
Indirect Object Recognition: Evidence for Associative Processes in Recognition Memory
}

\author{
Emma Whitt, Mark Haselgrove, and Jasper Robinson \\ University of Nottingham
}

\begin{abstract}
Rats' exploration of stimulus P (e.g., a domestic object) is reduced following either its direct exposure or its indirect exposure and is taken to indicate recognition memory. Procedures for demonstrating indirect object recognition involve an initial presentation of object $\mathrm{P}$ with stimulus $\mathrm{X}$ (and of an object Q with stimulus Y). On test, stimulus $\mathrm{X}$ is presented with objects $\mathrm{P}$ and Q and rats' exploration of Q exceeds their exploration of $\mathrm{P}$. One interpretation here is that the presentation of stimulus $\mathrm{X}$ on test associatively activates the memory of object $\mathrm{P}$, which diminishes exploration of $\mathrm{P}$ relative to $\mathrm{Q}$. It is possible, instead, that performance is simply the result of a novel pattern of stimulation generated by the unfamiliar combination of $\mathrm{X}$ and $\mathrm{Q}$. The authors modified this procedure to reduce the likelihood of such a process. Their procedure involved first the presentation of $\mathrm{PX}$ and $\mathrm{QY}$ before the presentation of stimulus $\mathrm{X}$ alone. During the test that followed, objects $\mathrm{P}$ and $\mathrm{Q}$ were presented but stimulus $\mathrm{X}$ was removed. The authors found that exploration of $\mathrm{Q}$ remained greater than that of $\mathrm{P}$ despite these modifications and discuss some theoretical implications of indirect, associative processes in recognition memory.
\end{abstract}

Keywords: recognition memory, object recognition, associative learning, habituation

In a typical object recognition experiment (e.g., Kivy, Earl, \& Walker, 1956; Ennaceur \& Delacour, 1988), rats are first presented with a pair of identical domestic objects (PP; e.g., two identical bottles) and are subsequently given a choice test in which the familiar P stimulus is presented with the novel Q stimulus (PQ; i.e., the original bottle and, e.g., a new jar). Sanderson and Bannerman (2011) recently suggested that performance on such tasks may be understood in terms of a model of memory proposed by Brandon, Vogel, and Wagner (2003; see also, Honey \& Good, 2000; Wagner, 1981). Here, stimuli are conceived of as collections of representational elements capable of existing in inactive or active states. Initial presentation of object $\mathrm{P}$ will promote $\mathrm{P}$ 's elements from inactivity into a primary state of activity (Al) provoking relatively strong responding (e.g., orienting, approach). Responding will diminish as the elements decay into a secondary (A2) state of activity before they, again, become inactive. Because elements are unable to enter their A1 state directly from their A2

This article was published Online First November 21, 2011.

Emma Whitt, Mark Haselgrove, and Jasper Robinson, School of Psychology, University of Nottingham, Nottingham, England.

Work reported here was funded by grants from the Biotechnology and Biological Sciences Research Council (BBSRC) to Mark Haselgrove (BB/ F01239X/1) and to Jasper Robinson (BB/C006283/1) and contributed to Emma Whitt's doctorate degree, which was funded by the School of Psychology, University of Nottingham and by a doctoral training award in integrative physiology from the BBSRC.

Procedures were authorized under United Kingdom law, Animals (Scientific Procedures) Act (1986).

Correspondence concerning this article should be addressed to Jasper Robinson, School of Psychology, University of Nottingham, University Park, Nottingham, NG7 2RD United Kingdom. E-mail: jasper.robinson@ nottingham.ac.uk state (e.g., Brandon et al., p. 9), the presentation of $\mathrm{P}$ on test (whose elements will be in their A2 states) provokes weaker approach behavior than the novel object, Q (whose elements can enter their A1 states directly from their inactive states). This has been the finding reported in a great variety of settings (see, e.g., Albasser, Davies, Futter, \& Aggleton, 2009; Hughes, 2007; Wan et al., 2001; see also Fagan, 1970).

A variant of this recognition memory task, reported by Dellu, Fauchey, Le Moal, and Simon (1997; see also Dix \& Aggleton, 1999; Eacott \& Gaffan, 2005; Honey, Good, \& Manser, 1998), is also explicable in terms of Brandon et al.'s (2003) model (Honey \& Good, 2000; Honey et al., 1998; Sanderson \& Bannerman, 2011). Rats were presented with both objects, P and Q, on separate trials and with each presented in a distinct context, respectively, $\mathrm{X}$ and Y. During the test, both objects were presented in context X, and rats were found to explore object $\mathrm{Q}$ more than object $\mathrm{P}$. Brandon et al.'s model will allow that excitatory associations may form between context cues and the objects (in particular, $\mathrm{X} \rightarrow \mathrm{P}$ and $\mathrm{Y} \rightarrow \mathrm{Q}$ ) during their pairing. And on test, context $\mathrm{X}$ elicits A2 activity in P's but not Q's elements. When a rat then encounters the objects, therefore, Q's elements will tend to enter their A1 states from their inactive states, provoking relatively great approach responding; however, because of their associative activation by $\mathrm{X}$, P's elements will tend to be in their A2 states where they provoke rather little responding and will be unable to enter their A1 states. Thus, Brandon et al.'s model can use direct (stimulus decay) and indirect (associatively activated) forms of A2 priming, respectively, to permit standard (e.g., Kivy et al., 1956) and contextual forms (e.g., Dix \& Aggleton, 1999) of recognition performance. Evidence supportive of this analysis has been reported in Pavlovian conditioning procedures (e.g., Kimble \& Ost, 1961; Knight, Lewis, \& Wood, 2011). 
Although the analysis of contextual recognition performance is consistent with Brandon et al.'s (2003) model, it is possible to understand it instead in terms of generalization decrement (e.g., Hull, 1943; Lovibond, Preston, \& Mackintosh, 1984). Here, the object and context are assumed to interact such that, for example, object Q will be perceived differently during the test in context $\mathrm{X}$ than it was during training in context Y. It may act, therefore, as a partially new stimulus, thus able to support approach behavior. Although such arguments can never really be dispelled (e.g., Hall, 1991), it is certainly possible to make arrangements to reduce any potential role for generalization decrement. Honey et al. (1998) reported one such strategy in which rats' orienting responses were measured to lights ( $\mathrm{P}$ and $\mathrm{Q})$ that were paired with auditory stimuli (respectively, $\mathrm{X}$ and $\mathrm{Y}$; i.e., $\mathrm{X} \rightarrow \mathrm{P}$ and $\mathrm{Y} \rightarrow \mathrm{Q}$ trials). Over the course of training, orienting responding elicited by the lights declined; but during testing, orienting recovered when a light was preceded by the alternative auditory stimulus (e.g., orienting to $\mathrm{P}$ was greater on $\mathrm{Y} \rightarrow \mathrm{P}$ trials than on $\mathrm{X} \rightarrow \mathrm{P}$ trials), a finding mirroring that of Dix and Aggleton (1999). This finding may be accommodated by Brandon et al.'s (2003) model, but an account based on generalization decrement seems less likely than in the example above because Honey et al. arranged for the auditoryvisual elements to be presented sequentially. Because there was no direct overlap between the stimuli, the kinds of interactions required for the generalization decrement argument seem relatively remote (e.g., Lovibond et al., 1984).

The purpose of these experiments (whose designs are summarized in the top panels of Figure 1 and Figure 3) was to investigate the prediction that indirect (i.e., associative) priming of an object's representation diminishes the approach responding it elicits. During Stage 1 of our experiments, rats were exposed to target objects $\mathrm{P}$ and $\mathrm{Q}$ that were presented simultaneously with, respectively, stimuli X and Y (objects in Experiment 1; wall inserts in Experiment 2 ), in order to permit the formation of excitatory associations
Stage 1

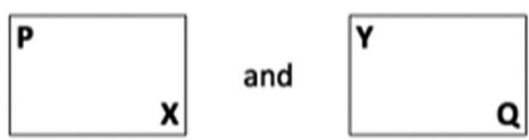

Stage 2

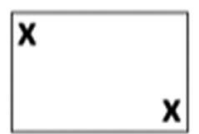

Test

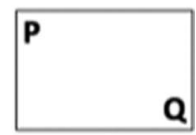

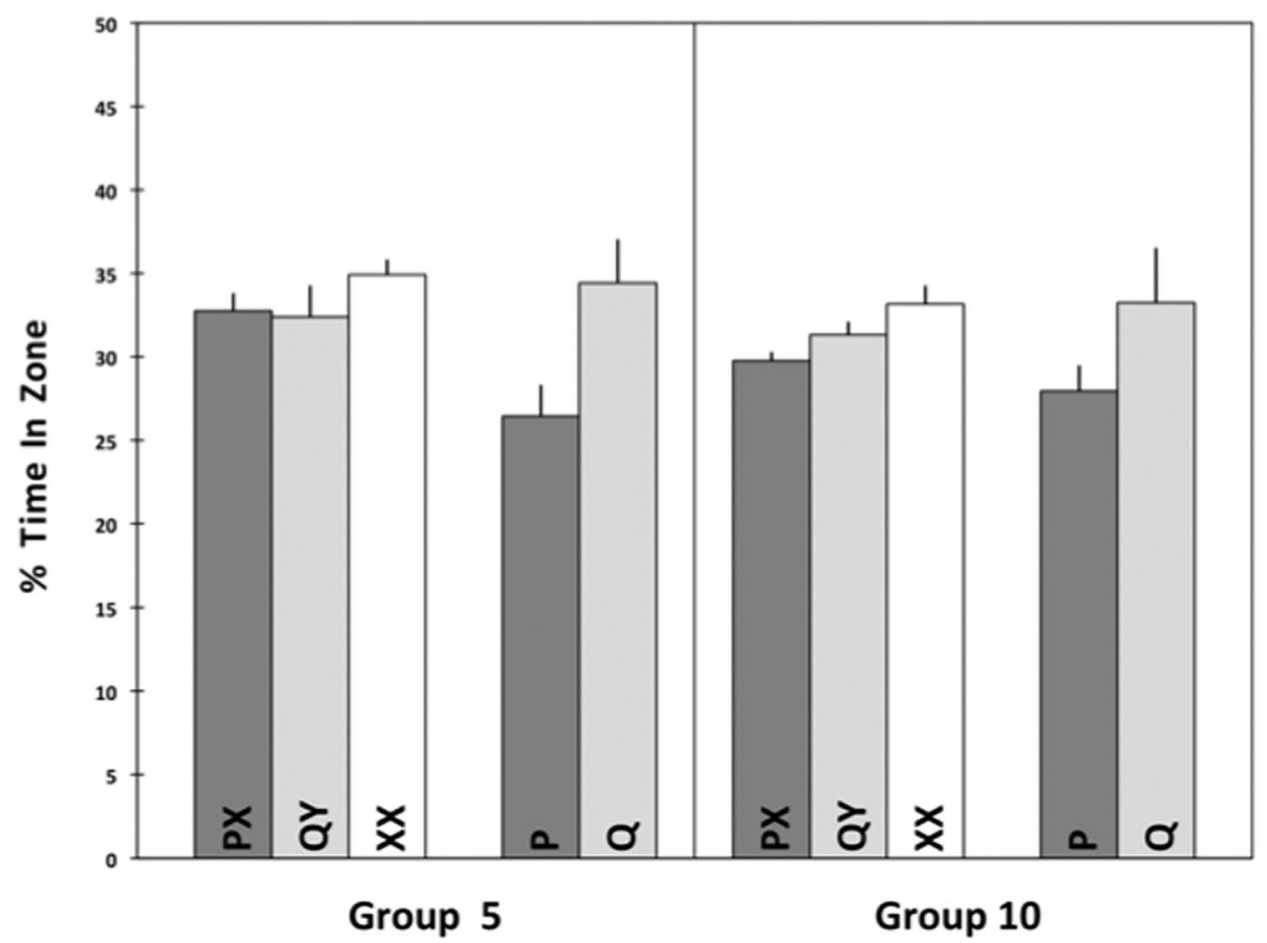

Figure 1. Upper panel: Schematic representation of Experiment 1. All rats were placed in an arena containing 2 domestic objects. Stage 1 comprised 2 trials. Objects $\mathrm{P}$ and $\mathrm{X}$ were presented on one of those trials; objects $\mathrm{Q}$ and $\mathrm{Y}$ on the other trial. During Stage 2, the arena contained only object X (XX). In the subsequent test, object $\mathrm{P}$ was presented with object Q. Treatments for Groups 5 and 10 were identical except that the durations of their Stage-1 and Stage-2 exposure was, respectively, $5 \mathrm{~min}$ and $10 \mathrm{~min}$. See the Method section for full details. Lower panel: Results of Experiment 1. Means and $1 S E$ of each mean from Experiment 1. Data for Groups 5 and 10 are, respectively, presented on the left and right. For both groups, the left trio of data represent the proportion (percentage) of time spent in zones during Stage 1 (PX and QY) and Stage $2(\mathrm{X})$; the right pair of data the proportion (percentage) of time spent in zones around the $\mathrm{P}$ and $\mathrm{Q}$ objects during the test. 
between them $(\mathrm{X} \rightarrow \mathrm{P}$ and $\mathrm{Y} \rightarrow \mathrm{Q})$. After this, rats were presented with $\mathrm{X}$ and then objects $\mathrm{P}$ and $\mathrm{Q}$. The anticipated result was that approach of Q should be greater than that of P (cf., Dix \& Aggleton, 1999). However, we sought to reduce the potential impact of any generalization decrement by presenting and removing X 10 min before test presentation of $\mathrm{P}$ and $\mathrm{Q}$. The presentation of $\mathrm{X}$ should indirectly activate stimulus $\mathrm{P}$ by associatively activating its elements into their A2 states. If so, it is possible that the standard finding will remain because presentation of $\mathrm{Q}$ on test will provoke its elements from their inactive states into their A1 state (eliciting relatively strong approach behavior) whereas presentation of $\mathrm{P}$ will do so only to the extent that its elements have decayed from their A2 state to their inactive state. Thus, the prediction of these experiments is that performance based on indirect recognition memory should be retained but that performance based on generalization decrement should be abolished.

\section{Experiment 1}

We set out to assess the possibility that recognition memory could be achieved, indirectly; that is, when the target stimulus, rather than being presented directly before test (e.g., Dellu et al., 1997; Dix \& Aggleton, 1999; Eacott \& Gaffan, 2005), is associatively activated (e.g., Honey et al., 1998; Sanderson \& Bannerman, 2011). Demonstrations in which exploratory responding is greater to an object presented in an apparatus that it has not before been exposed in (e.g., Dellu et al., 1997; Dix \& Aggleton, 1999; Eacott \& Gaffan, 2005) may be taken as evidence of the reality of indirect recognition memory (see also, e.g., Kimble \& Ost, 1961; Knight et al., 2011). However, performance is also explicable in terms of generalization decrement (e.g., Hull, 1943; Lovibond et al., 1984) and the results reported by Dellu et al., 1997; Dix and Aggleton, 1999; and Eacott and Norman, 2004, are especially prone to that suggestion because testing occurs with the target stimuli presented simultaneously with the apparatus cues with which they may perceptually interact. Experiment 1, whose design is depicted in the top panel of Figure 1, arranged for separation of the crucial stimulus, $\mathrm{X}$, and the target stimuli, $\mathrm{P}$ and $\mathrm{Q}$. We assumed that the nonsimultaneous presentation of $\mathrm{X}$ and $\mathrm{P}$ and $\mathrm{X}$ and $Q$ would minimize any effect of generalization decrement on performance but would permit associative, indirect processes. Thus, if the sole source of performance in such experiments is due to associative activation of $\mathrm{P}$ by $\mathrm{X}$, then responding to $\mathrm{Q}$ is expected to exceed that to $\mathrm{P}$; but if the sole source of performance is based only on generalization decrement, responding to $\mathrm{P}$ and to $\mathrm{Q}$ should be equivalent. Experiment 1 used domestic objects for stimuli $\mathrm{P}, \mathrm{Q}, \mathrm{X}$, and $\mathrm{Y}$.

\section{Method}

Subjects. Sixteen male Lister-hooded rats (Rattus norvegicus), supplied by Charles River (United Kingdom) served as experimental subjects. They were pair housed in cages in a room illuminated between 0700 and 1900. Rats' cages contained wood chip and paper bedding and a cardboard tube for environmental enrichment. On the day before the test, rats weighed between 320 and $380 \mathrm{~g}$, with a mean of $351 \mathrm{~g}$, and they had free access to food and water throughout the experiment. Rats had previously been exposed to auditory stimuli in conditioning chambers but were naive to the current apparatus and stimuli.
Apparatus. Four identically specified white, high-density polyethylene boxes served as arenas. Their molded construction resulted in curvature where the floor and walls met but the approximate dimensions were $60.0 \mathrm{~cm} \times 40.0 \mathrm{~cm} \times 45.0 \mathrm{~cm}$ high A sheet of white acrylic was fitted so as to provide a flat floor that perfectly met the walls. Multiple copies of four objects were used: (a) a blue, plastic bottle with height of $20.0 \mathrm{~cm}$ and circular base of $7.0 \mathrm{~cm}$ diameter; (b) a molded plastic bottle covered with black-and-white penguins with a height of $20.5 \mathrm{~cm}$ and maximum base dimensions of $9.0 \mathrm{~cm} \times 10.0 \mathrm{~cm}$; (c) a black glass hourglassshaped bottle with a height of $18.0 \mathrm{~cm}$ and a circular base of 6.5 $\mathrm{cm}$ diameter; (d) and a transparent glass mineral water bottle (23 $\mathrm{cm} \times 8.5 \mathrm{~cm})$. Objects could be secured to the floor using BluTac (Bostik, Leicester, United Kingdom) and were placed in opposite corners. A frame erected above the arena's open top centrally suspended a camera (Fire-I, Unibrain, Athens, Greece) $90.0 \mathrm{~cm}$ above floor level. Each camera's view (approximately $45^{\circ}$ arc) could be fed to a Microsoft Windows-based personal computer and included the complete floor but only the lower part of the four walls. A pair of light emitting diode (LED) lamps, $22 \mathrm{~cm}$ apart, center to center, flanked each camera and produced a floor-level illumination of $50 \mathrm{~lx}$. From the floor of the arena it was possible for a rat to see only a restricted view of the laboratory's ceiling; however, during operation of the lamps, the ceiling was not visible. The lamps were operated in all parts of the experiment. The personal computer was equipped with software (ANY-maze, Stoelting Co., Wood Dale, IL) that automatically tracked rats' head locations within the box. The software was also used to specify floor zones and to automatically record durations of rats' entry into a particular zone, which was taken as an index of its exploration of the stimulus within it. Comparison of automated and directly scored behavior is known to produce similar results (e.g., Silvers, Harrod, Mactutus, \& Booze, 2007). Aqueous ethanol solution $(70 \%, \mathrm{vol} / \mathrm{vol})$ and paper towels were used to clean the arenas and objects to reduce the likelihood of rat-odor cues influencing performance.

Procedure. A schematic representation of Experiment 1 is presented in the top panel of Figure 1. Rats were randomly assigned to one of two groups: Group $5(n=8)$ and Group $10(n=$ 8 ). Both groups were first given three daily 10-min exposures to an empty arena to promote habituation of nonspecific arena exploration. All experimental training was given on the following day. Stage 1 of training comprised two trials: For half of the rats in each group, stimuli $\mathrm{P}$ and $\mathrm{X}$ were presented together on the first Stage-1 trial, and stimuli $\mathrm{Q}$ and $\mathrm{Y}$ were presented together on the second Stage-1 trial; for the remainder the sequence of PX and QY presentation was reversed. For half of the rats in each group the blue bottle served as P and the penguin bottle served as Q; for the remainder of each group the roles of those objects were reversed. The black bottle and the transparent bottle served as X and Y and were counterbalanced in an identical fashion. The subgroups created by counterbalancing $\mathrm{P}$ and $\mathrm{Q}$ were represented equivalently in the subgroups created by counterbalancing $\mathrm{X}$ and $\mathrm{Y}$. Those four counterbalanced subgroups were represented equally in Group 5 and Group 10. The position of the objects within the arena was counterbalanced with the constraints: that objects P and Q did not occur in the same location on the two trials of Stage 1; and that objects $\mathrm{X}$ and $\mathrm{Y}$ did not occur in the same location on the two trials of Stage 1 (see top panel of Figure 1). Stage 2 followed Stage 1, 
and training consisted of a single trial in which two copies of object $\mathrm{X}$ were located in the two object zones. Testing occurred after Stage 2. During the test, all rats were presented with objects $\mathrm{P}$ and $\mathrm{Q}$, whose locations were the same as they had been during the Stage-1 trials. The Stage-1 and Stage-2 trial durations for Groups 5 and 10 were, respectively, 5 and $10 \mathrm{~min}$. The test stage was $5 \mathrm{~min}$ for both groups. Intervals between the three stages of the experiment were $10 \mathrm{~min}$ long. During these intervals rats were removed from the arenas and placed in their home cages while arenas and objects were cleaned and replaced. For rats in both groups, the two zones were located on two diagonally opposite corners of the arena floor. These zones were rectangular, measuring $13.5 \mathrm{~cm}$ by $12.5 \mathrm{~cm}$. The area of each zone was $169 \mathrm{~cm}^{2}$.

\section{Results and Discussion}

Results of Experiment 1 are summarized in the bottom panel of Figure 1. It is apparent that rats in Groups 5 and 10 spent similar proportions of trial time in the object zones to each other during Stage 1 and, of greater importance, similar proportions of time in PX zones as QY zones. This characterization of the data is supported with an analysis of variance (ANOVA), which yielded no main effects of PX versus QY, $F<1$, or group, $F(1,14)=3.52$, $p>.081, \eta_{\mathrm{p}}^{2}=.201$, and no interaction between those variables, $F<1$. Stage 2 entries to X's two zones were also similar in the two groups, $t(14)=1.23, p>.237, \eta_{\mathrm{p}}^{2}=.097$. Of central interest are the data from testing, which appear to indicate that, irrespective of group, rats tended to make a greater proportion of entries to the $\mathrm{Q}$ zone than to the $\mathrm{P}$ zone. This description of the data was confirmed by an ANOVA, which produced a main effect of $\mathrm{P}$ versus $\mathrm{Q}, F(1,14)=6.00, p<.027, \eta_{\mathrm{p}}^{2}=.300$, but neither a main effect of group nor an interaction between those variables, $F \mathrm{~s}<1$.

The results of Experiment 1 join those of related experiments (e.g., Dellu et al., 1997; Dix \& Aggleton, 1999; Eacott \& Gaffan, 2005) in showing an apparent role for associative processes in recognition memory, in which the stimulus $\mathrm{X}$ associatively activates the memory of $\mathrm{P}$ (priming its elements into their A2 state; Brandon et al., 2003; Honey et al., 1998; Sanderson \& Bannerman, 2011), subsequently reducing exploratory responding. Unlike previous experiments we arranged for nonsimultaneous presentation of $\mathrm{X}$ with $\mathrm{P}$ and with $\mathrm{Q}$, thereby making remote any role for generalization decrement (Hull, 1943). By elimination, therefore, our results appear best interpreted as an associatively based form of indirect recognition memory.

Before fully accepting that interpretation it is important to consider an alternative explanation of our results, which is best outlined by reference to the top panel of Figure 1. Notice that Stage 2 involves the presentation of copies of object $\mathrm{X}$ in two zones. One of the zones is that in which object $\mathrm{X}$ appeared during Stage 1 (bottom right, in the example in Figure 1), but the other is a new location for object X (top left). Because changes of location within an arena can restore approach behavior (e.g., Barker, Bird, Alexander, \& Warburton, 2007; Eacott \& Norman, 2004; Good, Barnes, Staal, McGregor, \& Honey, 2007), it is possible that rats tended to bias their approach toward the copy of $\mathrm{X}$ in its new location for longer than the copy of $\mathrm{X}$ in its original, Stage-1 location. Thus, on test, Q's location will have been explored relatively little and may provoke greater levels of exploratory behavior than P's location; that is, test performance could be based on (direct) location recognition rather than (indirect) object recognition. To examine this possibility, we looked again at Stage-2 data; the prediction from this alternative account being that rats' entries into the new $\mathrm{X}$ zone should exceed those of the original, Stage- 1 zone. The mean proportions of entries to the new and the original (Stage-1) X zones, for Group 5 were, respectively, 39.1\% $(S E M=0.03)$ and $30.8 \%(S E M=0.04)$; the corresponding data for Group 10 were $33.7 \%(S E M=0.03)$ and $32.6 \%(S E M=0.04)$. An ANOVA with group and zone variables yielded no main effect of group, $F(1,14)=1.52, p>.237, \eta_{\mathrm{p}}^{2}=.098$; nor effect of zone, $F<1, p>.344, \eta_{\mathrm{p}}^{2}=.064$; nor Group $\times$ Zone interaction, $F<$ $1, p>.461, \eta_{\mathrm{p}}^{2}=.039$. Separate tests on Groups 5 and 10 also failed to reveal differences in the new and original $\mathrm{X}$ zones, respectively, $t(7)=1.28, p>.247, \eta_{\mathrm{p}}^{2}=.214, t<1, p>.883$, $\eta_{\mathrm{p}}^{2}=.003$. We also inspected the paths that rats took during Stage 2. The paths from two randomly selected rats from each group are presented in Figure 2. It is evident that the density of the path is greater for the Group 10 rats than for the Group 5 rats (merely reflecting the differences in the durations of Stage 2 for the two groups) and that some regions of the arena floor were not entered (a result of differences in the sizes of the counterbalanced objects used). However, each rat's exploration of the two zones seemed similar and the paths seemed approximately symmetrical across the top left and bottom right halves of the arena's floor. There seems, therefore, to be little foundation for that alternative account based on Stage-2 differences in zone exploration.

We take our failure to detect differences in exploration here to be a feature of our procedure rather than an indication that successful demonstrations (e.g., Eacott \& Norman, 2004; Good et al., 2007) are to be doubted. In our survey of the literature, successful demonstrations permitted the position of an object to be encoded either by its relation to other objects in the arena (e.g., Barker et al., 2007; Dember, 1956; Good \& Hale, 2007; Good et al., 2007) or by its relation to objects beyond the arena's walls (e.g., Eacott \& Norman, 2004). In our own procedure, we attempted to eliminate such sources of performance (see Apparatus of Experiment 1) and were apparently successful.

\section{Experiment 2}

The results of Experiment 1 provided evidence of indirect recognition memory (e.g., Honey et al., 1998; Sanderson \& Bannerman, 2011; Wagner, 1981) that cannot be readily accommodated by alternative accounts based on generalization decrement (Hull, 1943). We noted also an alternative account based on differential exploration of the arena during Stage 2. In particular,

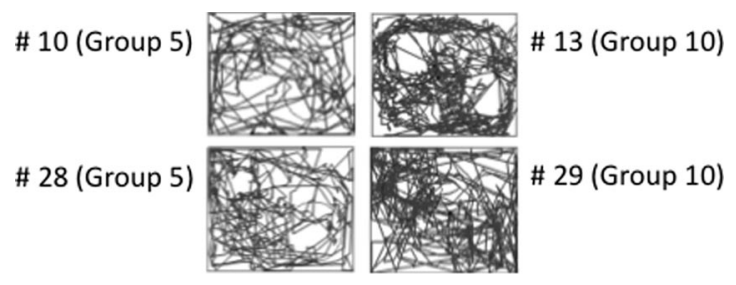

Figure 2. The paths taken by 4 rats during Stage 2 of Experiment 1 . The leftmost pair of paths is that of 2 rats (nos. 10 and 28) from Group 5; the rightmost pair of paths is that of 2 rats (nos. 13 and 29) from Group 10. 
Stage 1

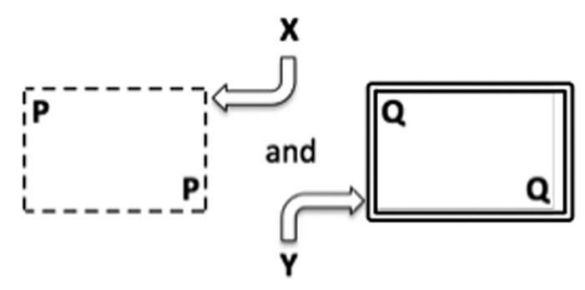

Stage 2

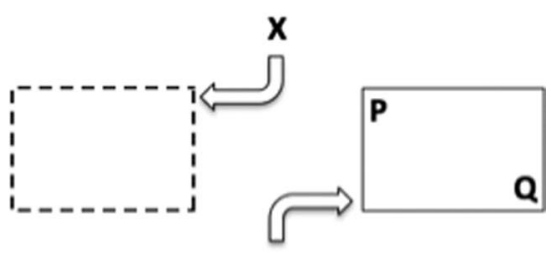

No wall inserts

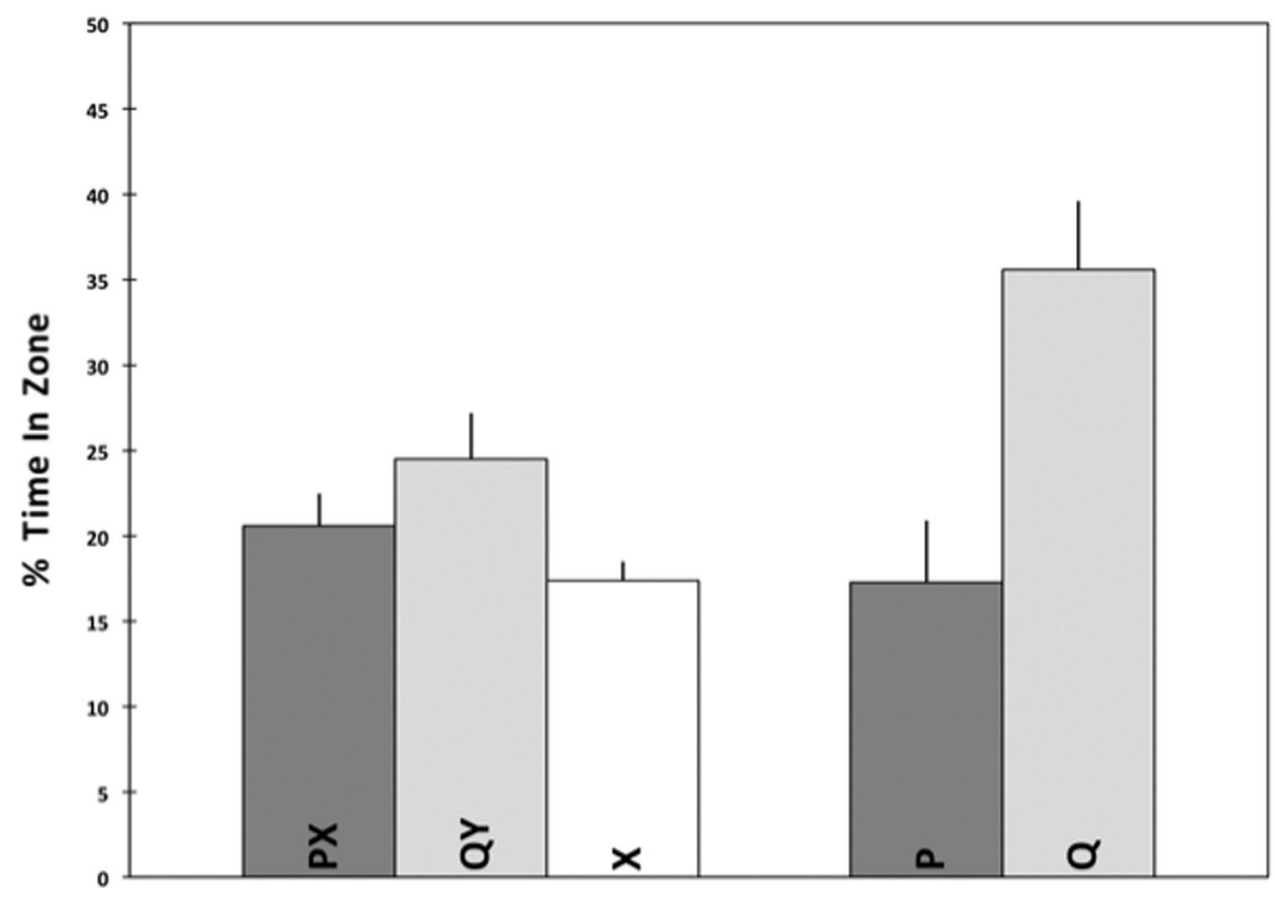

Figure 3. Upper panel: Schematic representation of Experiment 2. All rats were placed in an arena containing wall inserts and domestic objects. Stage 1 comprised 2 trials. Insert $X$ and a pair of object Ps were presented on one of those trials; insert Y and a pair of object Qs were presented on the other trial. During Stage 2, the arena contained insert $\mathrm{X}$ but no domestic objects. In the subsequent test, object $\mathrm{P}$ was presented with object $\mathrm{Q}$, but no wall inserts were presented. See the Method section for full details. Lower panel: Results of Experiment 2. Means and $1 S E$ of each mean from of Experiment 2. The left trio of data represents the proportion (percentage) of time spent in zones during Stage 1 (PX and QY) and Stage $2(\mathrm{X})$; the right pair of data the proportion (percentage) of time spent in zones around the $\mathrm{P}$ and $\mathrm{Q}$ objects during the test.

the Stage-2 presentation of stimulus $\mathrm{X}$ in a zone not used in Stage 1 could inflate approach toward the alternative zone in which object Q was located on test. Analysis of the Stage-2 data failed to support that account; however, the possibility remains that, although Stage-2 zone entries did not differ between X's new and its original (Stage 1) location, the new location of $\mathrm{X}$ modified processing in some other manner. For example, when in central (nonzone) regions of the arena, rats may have spent a disproportionate amount of time looking toward X's new location; or, although there was no difference in the amount of time spent in the new and original $\mathrm{X}$ zones, rats may have processed the new $\mathrm{X}$ zone more thoroughly through an increase in arousal provoked by the change. Either process would be undetectable but could affect test performance. For this reason, and to confirm the reliability and generality of Experiment 1's findings, we sought to replicate
Experiment 1 with a modified procedure in which such Stage-2 changes would not apply. The design of Experiment 2 is presented in the top panel of Figure 3. Experiment 2 followed the same logic as Experiment 1 except that the stimuli used as $\mathrm{X}$ and $\mathrm{Y}$ were wall inserts, which surrounded all four walls of each arena during Stages 1 and 2. Nonlocalized inserts were chosen to serve as $\mathrm{X}$ and $\mathrm{Y}$ to prevent biases in Stage-2 behavior that could influence test performance.

\section{Method}

Subjects. Sixteen rats of the same sex and strain as Experiment 1 were used. They were housed and kept as in Experiment 1. Rats weighed between 360 and $440 \mathrm{~g}$, with a mean weight of 393 g. Rats had previously been exposed to auditory stimuli in 
conditioning chambers, but were naive to the current apparatus and stimuli.

Apparatus. The arenas were those of Experiment 1. Multiple copies of a yellow, rubber duck (height $=9.0 \mathrm{~cm}$; maximum base dimensions $=9.0 \mathrm{~cm} \times 7.0 \mathrm{~cm})$ and a black-glass hourglassshaped bottle (height $=18.0 \mathrm{~cm}$; circular base $=6.5 \mathrm{~cm}$ diameter) served as stimuli. Wall inserts with two different patterns could be also be used as stimuli. These walls were made from medium density fiberboard covered in linoleum. The walls were $45.0 \mathrm{~cm}$ high, and when inside the box gave floor space of $42.0 \mathrm{~cm} \times 32.0$ $\mathrm{cm}$. Two different patterns were used. One consisted of a mosaic of blue squares, each square measuring $2.3 \mathrm{~cm}^{2}$. The squares' edges were $45^{\circ}$ from horizontal. The other linoleum pattern was composed of white, $272-\mathrm{cm}^{2}$ squares whose edges were either horizontal or vertical. A black, $16-\mathrm{cm}^{2}$ square was superimposed on each point where four white squares met. Each insert covered the whole of one of the shorter walls and half of both longer walls, and it was, therefore, possible to have either a single pattern or a pair of patterns in each arena. To the experimenter, differences in the squares' density, hue, and orientation made the insert distinctly different. However, in terms of the odor and tactile features of the linoleum, the inserts seemed identical.

Procedure. A schematic representation of Experiment 2 is presented in the top panel of Figure 3. Rats were first given exposure to an empty arena to promote habituation of nonspecific arena exploration. Experimental training was given on the following day. Stage 1 comprised two trials separated by a 10-min ITI. For half of the rats in each group, stimuli $\mathrm{P}$ and $\mathrm{X}$ were presented together on the first Stage-1 trial, and stimuli $\mathrm{Q}$ and $\mathrm{Y}$ were presented together on the second Stage-1 trial; for the remainder the sequence of PX and QY presentation was reversed. For half of the rats the black bottle served as $\mathrm{P}$, and the yellow duck served as Q; for the remainder of each group the roles of those objects was reversed. The blue-and-white wall inserts were identically counterbalanced in serving as $\mathrm{X}$ and $\mathrm{Y}$. The subgroups formed by counterbalancing $\mathrm{P}$ and $\mathrm{Q}$ were represented equivalently in the subgroups formed by counterbalancing $X$ and Y. Stage 2 followed Stage 1 and involved the placement of each rat into its arena, which contained insert $\mathrm{X}$ but no objects. Testing occurred after Stage 2. During testing rats were placed in an arena, which contained objects $\mathrm{P}$ and $\mathrm{Q}$ but contained no wall inserts. For half of the rats in each of the subgroups referred to above, object $P$ was located on the top left, and object Q was located on the bottom right, object zone; for the remainder, the objects received the reverse placement. All trials were $5 \mathrm{~min}$ in duration and were separated by an interval of $10 \mathrm{~min}$, during which rats were removed from the arenas and placed in their home cages while arenas and objects were cleaned and replaced. Two zones were located on two diagonally opposite corners of the arena floor. These zones were rectangular, measuring $13.5 \mathrm{~cm}$ by $12.5 \mathrm{~cm}$. The area of each insert zone was $169 \mathrm{~cm}^{2}$. Unspecified details were identical to those of Experiment 1.

\section{Results and Discussion}

One rat was excluded from analyses because of a marked side preference throughout the experiment, which left $N=15$. The Experiment 2 data are summarized in the lower panel of Figure 3. Rats spent a similar proportion of time in the PX and QY zones of
Stage $1, t(14)=1.08, p>.297, \eta_{\mathrm{p}}^{2}=.077$. The data of primary importance are those from testing, which indicate again that rats spent a greater proportion of time in the $\mathrm{Q}$ zone than the $\mathrm{P}$ zone, $t(14)=2.75, p<.016, \eta_{\mathrm{p}}^{2}=.352$. This finding joins that of Experiment 1 in demonstrating indirect recognition memory. To repeat, unlike similar findings (e.g., Dellu et al., 1997; Dix \& Aggleton, 1999; Eacott \& Gaffan, 2005), ours cannot be the result of the restoration of approach to novel stimulus patterns created by the simultaneous presentation of $\mathrm{X}$ and $\mathrm{Q}$ on test. We suggested that the results of Experiment 1 could be the result of undetected differences in the reception of the arena during Stage 2. But in Experiment 2, such differences were not possible. Our conclusion then is that this procedure provides reasonably strong evidence for the contribution of associative processes in recognition memory (see also, e.g., Kimble \& Ost, 1961; Knight et al., 2011). When taken as a whole, the results of the current experiments provide particular support for the account of object recognition that can be derived from Brandon et al.'s (2003) model (cf., Honey \& Good, 2000; Honey et al., 1998; Sanderson \& Bannerman, 2011).

\section{General Discussion}

We reasoned that extant demonstrations of indirect recognition memory (e.g., Dellu et al., 1997; Dix \& Aggleton, 1999; Eacott \& Gaffan, 2005) are also amenable to explanation in terms of generalization decrement caused by the simultaneous presentation of $\mathrm{X}$ with $\mathrm{Q}$ on test, for the first time. That source of performance was not possible in these experiments in which $\mathrm{X}$ was presented and removed before presentation of $\mathrm{P}$ and $\mathrm{Q}$. The prediction here was that indirect, associative activation of $\mathrm{P}$ should be possible, but that generalization decrement should not be. We suggested that the results of Experiment 1 could have been the result of the novel location of object X during Stage 2 (e.g., Eacott \& Norman, 2004; Good et al., 2007). However, analysis of Stage- 2 data failed to support that suggestion, and the design of Experiment 2 eliminated that possibility.

Our results are consistent with those of Sanderson and Bannerman (2011), who provided evidence for direct and indirect recognition processes of extramaze cues in mice (see also, Davis, 1970). Their interpretation is based on that of Brandon et al.'s (2003) model (see also Honey \& Good, 2000; Wagner, 1981) in which stimulus elements enter a secondary state of activity (A2) either via a direct path (following a primary state of activity, A1, that is permissible only from presentation of the stimulus) or through an indirect path (when associatively activated by a second stimulus). When in their A2 state, elements are unable to move to their A1 state without first passing through their inactive state. The dynamics of this system and the differences in the A1 and A2 states' ability to provoke responding are assumed to be the source of two processes of recognition, one direct and one indirect. Sanderson and Bannerman's main finding is that different optimal conditions exist for direct and indirect recognition, which they take as evidence of there being two sources of recognition. It is also apt to mention here demonstrations of relative recency in which two objects are presented in succession ( $\mathrm{Q}$ then $\mathrm{P}$ ) and the older object (Q) is explored more than the newer object $(\mathrm{P})$ when they are presented together for testing (e.g., Good et al., 2007). This finding is naturally amenable to an analysis based on Brandon et al.'s model. For example, a relatively large proportion of object Q's 
elements will have decayed from their A states to their inactive state by the time of testing. Presentation of Q at test may promote those elements into their A1 state, eliciting relatively vigorous approach behavior. However, as a consequence of its recent presentation, the majority of object P's elements remain in their A2 state where they cannot immediately enter their A1 state. They will, therefore, elicit only weak exploratory responding.

In addition to predicting that indirect recognition should be possible in this procedure, Brandon et al.'s (2003) model allows processes to occur on test. Let us consider first that no indirect recognition occurs at all. For example, perhaps object P's elements are activated into their A2 state by the presentation of stimulus $\mathrm{X}$ during Stage 2 but become inactive by the time of the test. Thus, on test, exploratory responding is based on the A1 activity of object $\mathrm{P}$ and Q's elements and the A2 activity in their respective, associated, stimuli $\mathrm{X}$ and $\mathrm{Y}$. Under these circumstances, of course, the levels of activity will be identical, and no discrimination is predicted. The only difference on test, given these particular assumptions, must be the result of stimulus X's elements residing in their A2 state during test. Brandon et al.'s model is explicitly blind to the path that elements take to reach their A2 states and so the fact that some of X's A2 activity is based on decay from A1 activity should not differentially alter the exploration of object $\mathrm{P}$ and Q that is supported. However, if the total number of X and Y's elements that are associatively activated into their A2 state by $\mathrm{P}$ and $\mathrm{Q}$ is less than the total number of elements that represent $\mathrm{X}$ and $\mathrm{Y}$, the decay-based $\mathrm{A} 2$ activation may increase the number of $\mathrm{X}$ elements in their A2 states to above that of the Y elements (see, e.g., Donegan, 1981). The natural prediction from this aspect of Brandon et al.'s model is, again, that test exploration of object $\mathrm{P}$ should, if anything, be greater than that of object Q- the opposite of what we report here. Of course, it is possible that the particular parameters we chose do not support such processes at all, or that their effects were weaker than the indirect recognition effect.

The indirect-activation account of performance occurs to us to be the most plausible account of the results of the current experiments, however, others are possible. We first consider an account based on stimulus intensity dynamism (Hull, 1949) — the finding that responding is related to stimulus intensity. During the test, objects $\mathrm{P}$ and $\mathrm{Q}$ might be affected by the persistent, directly activated memory of $\mathrm{X}$ from Stage 2, such that we might conceive their actual perception to be PX and QX. Furthermore, because each stimulus might be further elaborated by indirect activation of their Stage-1 associates, they may be perceived as PXX and QXY. In addition, it is feasible that the effect on intensity of a XY's addition to $\mathrm{Q}$ is greater than the addition of $\mathrm{XX}$ to $\mathrm{P}$. On the basis of these assumptions, stimulus Q could produce greater responding because it is, in effect, more intense than stimulus P. Although this account is possible, it must be acknowledged that it relies on a sequence of unsubstantiated assumptions and that the generality of stimulus intensity dynamism has been questioned (e.g., Gray, 1965).

Second, Rescorla (1974) has suggested that the stimulus memories that are the subject of associative learning can be updated after training. For example, Rescorla (1973) demonstrated that the repeated presentation of an unconditioned stimulus (the sounding of a klaxon) could reduce the conditioned suppression that was previously established to a light conditioned stimulus. Rescorla's (1974) explanation was that the memory of the unconditioned stimulus had been modified (i.e., the repeated presentation of the klaxon reduced its aversiveness and the memory of the klaxon was accordingly updated). It is possible to extend this interpretation to our indirect recognition demonstrations. We proposed earlier that the Stage-1 training in these experiments established $\mathrm{X} \rightarrow \mathrm{P}$ and $\mathrm{Y} \rightarrow \mathrm{Q}$; but $\mathrm{P} \rightarrow \mathrm{X}$ and $\mathrm{Q} \rightarrow \mathrm{Y}$ are also likely to form. The test presentation of objects $\mathrm{P}$ and $\mathrm{Q}$ might elicit similar levels of approach responding, but associative activation of, respectively, stimulus $\mathrm{X}$ and $\mathrm{Y}$ may supplement this. In addition, because stimulus X's exploratory strength may be weakened by its Stage-2 presentation (i.e., its stimulus memory has been modified), the net level of responding supported by $\mathrm{P}$ is less than that of $\mathrm{Q}$, whose associate, the memory of $\mathrm{Y}$, remains relatively strong. Rescorla (1974) gave little indication of the specific nature of the stimulus memory updating, but an appropriate solution is provided by the suggestion that habituation is simply the result of the decline in the efficiency of stimulus $\rightarrow$ response pathways (see, e.g., Horn, 1967; Mackintosh, 1987; Robinson, Sanderson, Aggleton, \& Jenkins, 2009). In this example, stimulus $\rightarrow$ stimulus $\rightarrow$ response pathways (i.e., $\mathrm{Q} \rightarrow \mathrm{Y} \rightarrow$ response and $\mathrm{P} \rightarrow \mathrm{X} \rightarrow$ response) would support rats' investigation of objects on test; however, the terminal $\mathrm{X} \rightarrow$ response path would have been fatigued by Stage- 2 presentation of $\mathrm{X}$, resulting in the reduced approach to $\mathrm{P}$ that was observed. Although this would adequately account for Rescorla's (1973) findings and our own demonstrations of indirect recognition, it does not easily apply to demonstrations of indirect object recognition in which stimulus $\mathrm{X}$ is present on test with objects $\mathrm{P}$ and $\mathrm{Q}$ (e.g., Dellu et al., 1997; Dix \& Aggleton, 1999; Eacott \& Gaffan, 2005).

The third alternative account to that of indirect recognition follows from the observation that, under certain circumstances, learning about the co-occurrence of events appears to result in the formation of an association that is susceptible to symmetrical extinction (e.g., Higgins \& Rescorla, 2004; see also, Aitken \& Dickinson, 2005). In this example, the presentation of stimulus $X$ during Stage 2 could result not only in the extinction of the stimulus $\mathrm{X} \rightarrow$ object $\mathrm{P}$ association but also in the extinction of an object $\mathrm{P} \rightarrow$ stimulus $\mathrm{X}$ association. The observed test discrimination is then predicted because object $\mathrm{Q}$ generates activity by its contact with the stimulus $Y$ representation (i.e., the operation of an intact $\mathrm{Q} \rightarrow \mathrm{Y}$ association), whereas object $\mathrm{P}$ is less well able to contact its associate, stimulus $\mathrm{X}$. It is notable that symmetrical extinction (and several other related phenomena) appears to occur in rats and pigeons only when presentation of the stimuli is arranged with a common onset and termination (see, e.g., Higgins \& Rescorla, 2004; Rescorla, 1981). In our procedure, rats' experience of stimulus $\mathrm{X}$ and object $\mathrm{P}$ would be quite unlike this: In Experiment 1, objects $\mathrm{P}$ and $\mathrm{X}$ were on opposite corners of the arena floor; and in Experiment 2, rats could sample stimulus X (an insert) without sampling object $\mathrm{P}$. Thus, although a symmetrical extinction account can, in principle, account for our findings, it falters on its reliance on parameters that were quite unlike those of our particular procedures.

We noted that the current experiments are consistent with the idea, central to Brandon et al.'s (2003) model, that two processes (direct and indirect activation) contribute to object recognition. However, they may be significant also because they stand in contrast to the predictions of two other models of stimulus representation. In order to explain effects associated with perceptual 
learning (e.g., Blair \& Hall, 2003a, 2003b), Hall (2003) has suggested that the two different pathways (direct and indirect) by which a stimulus representation can be activated have different consequences for the changes in the effectiveness, or sensitivity, of that representation. Direct activation (as a consequence of physically presenting a stimulus) results in habituation-that is to say a reduction in the ability of that stimulus to subsequently evoke behavior. Indirect activation (as a consequence of associatively activating a stimulus representation) results in the opposite effect, antihabituation, in which the effectiveness, or sensitivity, of a stimulus is restored. The model proposed by McLaren and Mackintosh (2000) makes the same prediction. According to McLaren and Mackintosh's analysis of the effects of exposure on stimulus processing, the presentation of a stimulus (i.e., direct activation) will result in the strengthening of associations among the elements responsible for its representation - the effect of which is to reduce the salience of that stimulus. However, associative (i.e., indirect) activation of a stimulus representation should have the opposite effect. Under these circumstances any associations among the elements that represent the stimulus will be extinguishedenhancing the salience of that stimulus. The results of the experiments described here are consistent with neither Hall's (2003) nor McLaren and Mackintosh's (2000) analyses: The associative activation of object $\mathrm{P}$ diminished (rather than enhanced) its effectiveness in supporting its subsequent exploration during testing. Note also that this indirect form of object recognition is of the same direction as that seen in standard, direct object recognition (e.g., Kivy et al., 1956; Ennaceur \& Delacour, 1988), rather than the opposite direction that Hall (2003) and McLaren and Mackintosh (2000) required (see also, Dwyer \& Honey, 2007).

Variations on object recognition tasks in rats have been used to support the notion of their having episodic-like memory. For example, Eacott, Easton, and Zinkivskay (2005) gave rats multiple presentations of two objects (e.g., $\mathrm{P}$ and $\mathrm{Q}$ ), each being presented simultaneously in one of two arenas (e.g., $\mathrm{X}$ and $\mathrm{Y}$ ). Over the course of multiple successive trials, object $\mathrm{P}$ would be presented, for some rats, on the leftmost side of arena $X$ while object $Q$ was presented on the rightmost side of arena $X$ (i.e., X: PIQ). On trials with arena $\mathrm{Y}$, the position of object $\mathrm{P}$ and $\mathrm{Q}$ was reversed (i.e., $\mathrm{Y}$ : $\mathrm{Q} \mid \mathrm{P})$. The floor of the arenas was $\mathrm{E}$ shaped, with the start arm being in the center and the choice arms being to the left and right. During initial training, the objects were positioned at the entrance to each choice arm-where rats could see them from the arena's start arm; but, during subsequent training, objects were positioned at the ends of the choice arms, so that they could not be seen from the start arm. Interpolated throughout training were exposures to only a single object, which was given in the home cage rather than in either of the arenas. The authors found that, over the trials that followed the single-object exposure, rats turned toward the position of the object that had not just been exposed. In this example (X: PIQ, Y: Q|P), exposure to object $\mathrm{P}$ in the home cage would encourage a rightward turn (toward $\mathrm{Q}$ ) when the next trial was in arena $X$. Of greatest significance here is the fact that above-chance performance was found irrespective of the visibility of the objects.

Eacott et al. (2005) interpreted their findings as evidence of separable familiarity and recollection components of episodic-like memory (see, e.g., Squire, Wixted, \& Clark, 2007). That is, with the objects visible, performance could be based solely on rats having a sense of familiarity with the single object encountered in the home cage immediately before the trial; but with the objects hidden from sight, rats would need to recall the training information for accurate performance. However, it is also possible to understand Eacott et al.'s findings in terms of the Brandon et al. (2003) model we have suggested as an explanation of our current results. The apparent familiarity-based performance (visible objects) may reflect differences in the activity states of the representations of the two objects on test (a relatively great proportion of the recently presented object's elements will be in their A2 state, provoking little approach i.e., directly activated recognition memory). The apparent recollection-based performance (invisible objects) may reflect indirect recognition memory. We know from the rats' performance that the left and right sides and the two types of arena are discriminable. Thus, we might expect object $\mathrm{P}$ to acquire two associates (e.g., one with the leftmost side of arena $\mathrm{X}[\mathrm{P} \rightarrow$ $L X]$ and another with the rightmost side of arena $\mathrm{Y}[\mathrm{P} \rightarrow R Y]$ ), with $\mathrm{Q}$ acquiring two different associates $(\mathrm{Q} \rightarrow \mathrm{LY}$ and $\mathrm{Q} \rightarrow \mathrm{RX})$. Presentation of, for example, object $\mathrm{P}$ in the home cage will indirectly activate LX and RYs' representational elements into their A2 states. If the following trial involves placement into arena $\mathrm{X}$ the unprimed RX stimulus will elicit greater approach behavior than the primed LX stimulus; but when the following trial involves placement into arena $\mathrm{Y}$, the unprimed LY stimulus can elicit greater approach than the primed RY (cf., Honey \& Good, 2000).

Although certain versions of alternative accounts could not be fully eliminated, these experiments provide evidence for a form of indirect object recognition (see also, e.g., Dellu et al., 1997; Dix \& Aggleton, 1999; Eacott \& Gaffan, 2005). It is notable that these accounts all relied on associative processes. We focused on the associative analysis for object recognition that can be derived from the theory proposed by Brandon et al. (2003). However, the general idea that stimulus representations are diminished when associatively activated is not unique to this model, it forms the basis for many theories of learning that require variations in stimulus processing with experience (e.g., Esber \& Haselgrove, 2011; Rescorla \& Wagner, 1972). Our use of separate stages in a variant of the procedure described by Dellu et al. (1997) and Dix and Aggleton (1999) enabled us to conclude that accounts of indirect object recognition based on generalization decrement were likely to be incomplete. We noted (see also, e.g., Honey \& Good, 2000; Sanderson \& Bannerman, 2011) that such demonstrations are naturally consistent with Brandon et al.'s (2003) model but appear to challenge McLaren and Mackintosh's (2000) model.

\section{References}

Aitken, M. R. F., \& Dickinson, A. (2005). Simulations of a modified SOP model applied to retrospective revaluation of human causal learning. Learning \& Behavior, 33, 147-159. doi:10.3758/BF03196059

Albasser, M. M., Davies, M., Futter, J. E., \& Aggleton, J. P. (2009). Magnitude of the object recognition deficit associated with perirhinal cortex damage in rats: Effects of varying the lesion extent and the duration of the sample period. Behavioral Neuroscience, 123, 115-124. doi: $10.1037 / \mathrm{a} 0013829$

Barker, G. R. I., Bird, F., Alexander, V., \& Warburton, E. C. (2007). Recognition memory for objects, place, and temporal order: A disconnection analysis of the role of the medial prefrontal cortex and perirhinal cortex. Journal of Neuroscience, 27, 2948-2957. doi:10.1523/ JNEUROSCI.5289-06.2007

Blair, C. A. J., \& Hall, G. (2003a). Changes in stimulus salience as a result 
of stimulus preexposure: Evidence from aversive and appetitive testing procedures. Learning \& Behavior, 31, 185-191. doi:10.3758/ BF03195981

Blair, C. A. J., \& Hall, G. (2003b). Perceptual learning in flavor aversion: Evidence for learned changes in stimulus effectiveness. Journal of Experimental Psychology: Animal Behavior Processes, 29, 39-48. doi: 10.1037/0097-7403.29.1.39

Brandon, S. E., Vogel, E. H., \& Wagner, A. R. (2003). Stimulus representation in SOP: I Theoretical rationalization and some implications. Behavioural Processes, 62, 5-25. doi:10.1016/S0376-6357(03)00016-0

Davis, M. (1970). Effects of interstimulus interval length and variability on startle-response habituation in the rat. Journal of Comparative and Physiological Psychology, 72, 177-192. doi:10.1037/h0029472

Dellu, F., Fauchey, V., Le Moal, M., \& Simon, H. (1997). Extension of a new two-trial memory task in the rat: Influence of environmental context on recognition processes. Neurobiology of learning and memory, 67, 112-120. doi:10.1006/nlme.1997.3746

Dember, W. N. (1956). Response by the rat to environmental change. Journal of Comparative and Physiological Psychology, 49, 93-95. doi: 10.1037/h0045411

Dix, S. L., \& Aggleton, J. P. (1999). Extending the spontaneous preference test of recognition: Evidence of object-location and object-context recognition. Behavioural Brain Research, 99, 191-200. doi:10.1016/ S0166-4328(98)00079-5

Donegan, N. H. (1981). Priming-produced facilitation or diminution of responding to a Pavlovian unconditioned stimulus. Journal of Experimental Psychology: Animal Behavior Processes, 7, 295-312. doi: 10.1037/0097-7403.7.4.295

Dwyer, D. M., \& Honey, R. C. (2007). The effects of habituation training on compound conditioning are not reversed by an associative activation treatment. Journal of Experimental Psychology: Animal Behavior Processes, 33, 185-190. doi:10.1037/0097-7403.33.2.185

Eacott, M. J., Easton, A., \& Zinkivskay, A. (2005). Recollection in an episodic-like memory task in the rat. Learning \& Memory, 12, 221-223. doi: $10.1101 / \mathrm{lm} .92505$

Eacott, M. J., \& Gaffan, E. A. (2005). The roles of perirhinal cortex, postrhinal cortex, and the fornix in memory for objects, contexts, and events in rats. Quarterly Journal of Experimental Psychology, 58B, 202-217.

Eacott, M. J., \& Norman, G. (2004). Integrated memory for object, place, and context in rats: A possible model of episodic-like memory? Journal of Neuroscience, 24, 1948-1953. doi:10.1523/JNEUROSCI.297503.2004

Ennaceur, A., \& Delacour, J. (1988). A new one-trial test for neurobiological studies of memory in rats. 1: Behavioral data. Behavioural Brain Research, 31, 47-59. doi:10.1016/0166-4328(88)90157-X

Esber, G. R., \& Haselgrove, M. (2011). Reconciling the influence of predictiveness and uncertainty on stimulus salience: A model of attention in associative learning. Proceedings of the Royal Society B, 278, 2553-2561. doi:10.1098/rspb.2011.0836

Fagan, J. F. (1970). Memory in the infant. Journal of Experimental Child Psychology, 9, 217-226. doi:10.1016/0022-0965(70)90087-1

Good, M. A., Barnes, P., Staal, V., McGregor, A., \& Honey, R. C. (2007). Context- but not familiarity-dependent forms of object recognition are impaired following excitotoxic hippocampal lesions in rats. Behavioral Neuroscience, 121, 218-223. doi:10.1037/0735-7044.121.1.218

Good, M. A., \& Hale, G. (2007). The "Swedish" mutation of the amyloid precursor protein APPswe) dissociates components of object-location memory in aged Tg2576 mice. Behavioral Neuroscience, 121, 11801191. doi:10.1037/0735-7044.121.6.1180

Gray, J. A. (1965). Stimulus intensity dynamism. Psychological Bulletin, 63, 180-196. doi:10.1037/h0021700

Hall, G. (1991). Perceptual and associative learning. Oxford, England: Clarendon Press. doi:10.1093/acprof:oso/9780198521822.001.0001
Hall, G. (2003). Learned changes in the sensitivity of stimulus representations: Associative and nonassociative mechanisms. Quarterly Journal of Experimental Psychology, 56B, 43-55.

Higgins, T., \& Rescorla, R. A. (2004). Extinction and retraining of simultaneous and successive flavor conditioning. Learning \& Behavior, 32, 213-219. doi:10.3758/BF03196022

Honey, R. C., \& Good, M. (2000). Associative modulation of the orienting response: Distinct effects revealed by hippocampal lesions. Journal of Experimental Psychology, 26, 3-14.

Honey, R. C., Good, M., \& Manser, K. L. (1998). Negative priming in associative learning: Evidence from a serial-habituation procedure. Journal of Experimental Psychology: Animal Behavior Processes, 24, 229 237. doi:10.1037/0097-7403.24.2.229

Horn, G. (1967, August 12). Neuronal mechanisms of habituation. Nature, 215, 707-711. doi:10.1038/215707a0

Hughes, R. N. (2007). Rats' responsiveness to tactile changes encountered in the dark, and the role of mystacial vibrissae. Behavioural Brain Research, 179, 273-280. doi:10.1016/j.bbr.2007.02.023

Hull, C. L. (1943). Principles of behavior. New York, NY: AppletonCentury.

Hull, C. L. (1949). Stimulus intensity dynamism (V) and stimulus generalization. Psychological Review, 56, 67-76. doi:10.1037/h0058051

Kimble, G. A., \& Ost, J. W. P. (1961). A conditioned inhibitory process in eyelid conditioning. Journal of Experimental Psychology, 61, 150-156. doi: $10.1037 / \mathrm{h} 0044932$

Kivy, P. N., Earl, R. W., \& Walker, E. L. (1956). Stimulus context and satiation. Journal of Comparative and Physiological Psychology, 49 90-92. doi:10.1037/h0040984

Knight, D. C., Lewis, E. P., \& Wood, K. H. (2011). Conditioned diminution of the unconditioned skin conductance response. Behavioral Neuroscience, 125, 626-631. doi:10.1037/a0024324

Lovibond, P. F., Preston, G. C., \& Mackintosh, N. J. (1984). Context specificity of conditioning, and latent inhibition. Journal of Experimental Psychology: Animal Behavior Processes, 10, 360-375. doi:10.1037/ 0097-7403.10.3.360

Mackintosh, N. J. (1987). Neurobiology, psychology and habituation. Behavioural Research and Therapy, 2, 81-97. doi:10.1016/00057967(87)90079-9

McLaren, I. P. L., \& Mackintosh, N. J. (2000). An elemental model of associative learning: I. Latent inhibition and perceptual learning. Animal Learning \& Behavior, 28, 211-246. doi:10.3758/BF03200258

Rescorla, R. A. (1973). Effect of US habituation following conditioning. Journal of Comparative and Physiological Psychology, 82, 137-143. doi:10.1037/h0033815

Rescorla, R. A. (1974). Effect of inflation of the unconditioned stimulus value following conditioning. Journal of Comparative and Physiological Psychology, 86, 101-106. doi:10.1037/h0035964

Rescorla, R. A. (1981). Simultaneous associations. In P. Harzem \& M. Zeiler (Eds.), Advances in analysis of behavior (Vol. 2; pp. 47-80). New York, NY: Wiley.

Rescorla, R. A., \& Wagner, A. R. (1972). A theory of Pavlovian conditioning: Variations in the effectiveness of reinforcement and nonreinforcement. In A. H. Black \& W. F. Prokasy (Eds.), Classical conditioning II: Current research and theory (pp. 64-99). New York, NY: Appleton-Century-Crofts.

Robinson, J., Sanderson, D. J., Aggleton, J. A., \& Jenkins, T. A. (2009). Suppression to visual, auditory, and gustatory stimuli habituates normally in rats with excitotoxic lesions of the perirhinal cortex. Behavioral Neuroscience, 123, 1238-1250. doi:10.1037/a0017444

Sanderson, D. J., \& Bannerman, D. M. (2011). Competitive short-term and long-term memory processes in spatial habituation. Journal of Experimental Psychology: Animal Behavior Processes, 37, 189-199. doi: 10.1037/a0021461

Silvers, J. M., Harrod, S. B., Mactutus, C. F., \& Booze, R. M. (2007). 
Automation of the novel object recognition task for use in adolescent rats. Journal of Neuroscience Methods, 166, 99-103. doi:10.1016/ j.jneumeth.2007.06.032

Squire, L. R., Wixted, J. T., \& Clark, R. E. (2007). Recognition memory and the medial temporal lobe: A new perspective. Nature Neuroscience, 8, 872-884. doi:10.1038/nrn2154

Wagner, A. R. (1981). SOP: A model of automatic memory processing in animal behavior. In N. E. Spears \& R. R. Miller (Eds.). Information processing in animals: Memory mechanisms (pp. 5-47). Hillsdale, NJ: Erlbaum.
Wan, H., Warburton, E. C., Kuśmierek, P., Aggleton, J. P., Kowalska D. M., \& Brown, M. W. (2001). Fos imaging reveals differential neuronal activation of areas of rat temporal cortex by novel and familiar sounds. European Journal of Neuroscience, 14, 118-124. doi:10.1046/ j.0953-816x.2001.01625.x

Received June 17, 2011

Revision received August 25, 2011 Accepted September 13, 2011

\section{Members of Underrepresented Groups: Reviewers for Journal Manuscripts Wanted}

If you are interested in reviewing manuscripts for APA journals, the APA Publications and Communications Board would like to invite your participation. Manuscript reviewers are vital to the publications process. As a reviewer, you would gain valuable experience in publishing. The P\&C Board is particularly interested in encouraging members of underrepresented groups to participate more in this process.

If you are interested in reviewing manuscripts, please write APA Journals at Reviewers@apa.org. Please note the following important points:

- To be selected as a reviewer, you must have published articles in peer-reviewed journals. The experience of publishing provides a reviewer with the basis for preparing a thorough, objective review.

- To be selected, it is critical to be a regular reader of the five to six empirical journals that are most central to the area or journal for which you would like to review. Current knowledge of recently published research provides a reviewer with the knowledge base to evaluate a new submission within the context of existing research.

- To select the appropriate reviewers for each manuscript, the editor needs detailed information. Please include with your letter your vita. In the letter, please identify which APA journal(s) you are interested in, and describe your area of expertise. Be as specific as possible. For example, "social psychology" is not sufficient-you would need to specify "social cognition" or "attitude change" as well.

- Reviewing a manuscript takes time (1-4 hours per manuscript reviewed). If you are selected to review a manuscript, be prepared to invest the necessary time to evaluate the manuscript thoroughly. 